\title{
DYNKIN GAMES WITH HETEROGENEOUS BELIEFS
}

\author{
ERIK EKSTRÖM ${ }^{1}$, KRISTOFFER GLOVER AND MARTA LENIEC
}

\begin{abstract}
We study zero-sum optimal stopping games (Dynkin games) between two players who disagree about the underlying model. In a Markovian setting, a verification result is established showing that if a pair of functions can be found that satisfies some natural conditions, then a Nash equilibrium of stopping times is obtained, with the given functions as the corresponding value functions. In general, however, there is no uniqueness of Nash equilibria, and different equilibria give rise to different value functions. As an example, we provide a thorough study of the game version of the American call option under heterogeneous beliefs. Finally, we also study equilibria in randomized stopping times.
\end{abstract}

\section{INTRODUCTION}

There is an extensive literature on zero-sum optimal stopping games (Dynkin games) under homogeneous beliefs, see [2], [4] and [16] for some classical references. These games have later found applications for example in mathematical finance, see [13], which motivated further studies, see for example [1], [5], [6], [7], [14] and [19]. Other more recent contributions study various modifications of the zero-sum optimal stopping game. For example, in [18] it is shown that the game has a value under very general conditions on the payoff processes if randomized strategies are used. Furthermore, [8] studies a game with randomized strategies and asymmetric information about the payoff functions and in [15], a game with asymmetric information about the time horizon is studied. Another natural extension is to nonzero-sum games. Here a classical reference is [3], in which the existence of a Nash equilibrium in a Markovian setting was reduced to the existence of a solution of a variational inequality. More recently, [10] provided very general conditions under which a Nash equilibrium in stopping times exists for nonzero-sum games in a martingale setting.

A common assumption in all the above references is that the players agree about the distributional properties of the underlying stochastic process. In many situations, however, it is natural that the two players disagree about the model for the underlying. For example, the buyer of a call option would

Date: August 27, 2015.

2000 Mathematics Subject Classification. Primary 60G40; Secondary 91G20.

Key words and phrases. Dynkin games; heterogeneous beliefs; multiple Nash equilibria; optimal stopping theory.

${ }^{1}$ Support from the Swedish Research Council (VR) is gratefully acknowledged. 
typically estimate the drift of the underlying asset higher than would the seller. Of course, standard hedging arguments show that to avoid arbitrage possibilities, pricing should be done in a risk-neutral setting. However, if the players are not interested in hedging the risk away, or even prohibited from trading in the underlying, then their estimate of the drift is an important consideration. In the current article we study zero-sum optimal stopping games under heterogeneous beliefs about the distributional properties of the underlying stochastic process. More precisely, we study the case in which the underlying process is a diffusion, and the two players estimate the drift of the diffusion differently. Such a situation may arise, for example, from the use of different calibration methods, or simply from different views about the future (for example due to overconfidence). We also allow the players to apply different discount rates for valuation, caused, for example, by differing attitudes towards risk, or by different funding costs. However, an important assumption we make is that the players agree to disagree in the sense that each player has full information about the other player's beliefs. In this way, we avoid additional technical difficulties associated with the inference of beliefs.

It should be noted that the impact of heterogeneous beliefs on more general asset pricing models has been well studied in the existing literature (see, for example, the surveys in [9] and [11]). However, such work has almost exclusively focused on general equilibrium models in which a single asset price is established via a market clearing condition (i.e., that supply equals demand). To our knowledge, this is the first paper that considers the effect of diverse beliefs on the equilibrium outcome of an optimal stopping game.

In general, there will not exist a consensus about the value of the optimal stopping game under heterogeneous beliefs. Indeed, given a Nash equilibrium of stopping times, the seller and the buyer will estimate the distributional properties of the expected payoff differently, and thus the seller's value function and the buyer's value function will disagree. Moreover, unlike the case of zero-sum games under homogeneous beliefs, different Nash equilibria will lead to different value functions. In a Markovian setting, we provide a verification result that shows that if two given functions satisfy a set of natural conditions, then a Nash equilibrium is obtained such that the two functions are the values for the players. We then use the verification result to determine Nash equilibria for the game version of the American call option under heterogeneous beliefs when the underlying diffusion is a geometric Brownian motion. In particular, we show that under some parameter regimes there exist multiple equilibria, with different associated value functions.

We also include a study of optimal stopping games under heterogeneous beliefs allowing for randomized strategies. A verification result is provided in this setting, and it is used in a study of the game version of the American call option to find another Nash equilibrium. While the Nash equlibria in 
terms of stopping times are typically hitting times, the equlibria in randomized stopping times may be described as intensity based in the sense that the players exercise with certain level-dependent intensities. This leads to potentially non-smooth value functions.

The paper is organised as follows. In Section 2 we introduce the model for heterogeneous beliefs and the optimal stopping game, and we specify conditions under which there exists a Nash equilibrium. In Section 3 we provide the verification theorem, which is used in Section 4 in the study of the game version of the American call option. Finally, in Section 5 a verification result for randomized strategies is given, and it is applied to the game version of the American call option.

\section{Heterogeneous beliefs and NAsh equilibria}

We study an optimal stopping game between two players, Player 1 and Player 2, in which the players disagree about the distributional properties of the underlying stochastic process. To describe the setting mathematically, let $\left(\Omega, \mathcal{F}, \mathbb{F}, \mathbb{P}^{1}\right)$ be a filtered probability space, where $\mathbb{F}=\left(\mathcal{F}_{t}\right)_{t>0}$ is the completion of the filtration generated by a Brownian motion $W^{1}$. Player 1 models the underlying stochastic process $X$ as an $\mathbb{F}$-adapted diffusion process driven by $W^{1}$ with drift $\mu_{1}$, i.e.,

$$
d X_{t}=\mu_{1}\left(X_{t}\right) d t+\sigma\left(X_{t}\right) d W_{t}^{1}, \quad X_{0}=x,
$$

where $x$ is a positive deterministic constant and the two functions $\mu_{1}: \mathbb{R} \rightarrow$ $\mathbb{R}$ and $\sigma: \mathbb{R} \rightarrow(0, \infty)$ satisfy the standard Lipschitz condition so that a unique, non-exploding, strong solution exists.

Let $\mu_{2}$ be another Lipschitz continuous function, and let

$$
\eta_{t}=\frac{\mu_{2}\left(X_{t}\right)-\mu_{1}\left(X_{t}\right)}{\sigma\left(X_{t}\right)}
$$

We assume that the process $Z$ defined by

$$
d Z_{t}=\eta_{t} Z_{t} d W_{t}^{1}, \quad Z_{0}=1
$$

is a $\left(\mathbb{P}^{1}, \mathbb{F}\right)$-martingale (e.g., if the functions $\mu_{1}, \mu_{2}$ and $\sigma$ are such that $\eta$ satisfies the Novikov condition). Player 2 uses a measure $\mathbb{P}^{2}$ under which the process

$$
W_{t}^{2}=W_{t}^{1}-\int_{0}^{t} \eta_{s} d s
$$

is a Brownian motion. Hence Player 2 models $X$ as an $\mathbb{F}$-adapted diffusion process driven by $W^{2}$ with drift $\mu_{2}$, i.e.,

$$
d X_{t}=\mu_{2}\left(X_{t}\right) d t+\sigma\left(X_{t}\right) d W_{t}^{2}, \quad X_{0}=x .
$$

Remark The existence of a probability space with two probability measures as described above is guaranteed by $[12$, Corollary 3.5 .2$]$. The measures $\mathbb{P}^{1}$ and $\mathbb{P}^{2}$ are equivalent when restricted to $\mathcal{F}_{T}, T<\infty$, but not necessarily as measures on $\mathcal{F}$. Also note that we assume that the players agree about the volatility $\sigma$. In fact, since the process $X$ is fully observable, the instantaneous 
volatility is also observable, and therefore different views on the volatility would be in contradiction with the equivalence of the two measures for finite times.

Player 1 and Player 2 choose stopping times $\tau_{1}$ and $\tau_{2}$, respectively, and at $\tau_{1} \wedge \tau_{2}:=\min \left\{\tau_{1}, \tau_{2}\right\}$ Player 1 receives the amount

$$
G_{1}\left(X_{\tau_{1}}\right) 1_{\left\{\tau_{1} \leq \tau_{2}\right\}}+G_{2}\left(X_{\tau_{2}}\right) 1_{\left\{\tau_{2}<\tau_{1}\right\}}
$$

from Player 2, where $G_{1}$ and $G_{2}$ are two given continuous functions satisfying $G_{1} \leq G_{2}$. In (2), and in all similar situations below, we use the convention that, for any function $f$ and any stopping time $\tau, f\left(X_{\tau}\right)=0$ on the set $\{\tau=\infty\}$. Using a constant discount rate $r_{i}>0$, today's value of the future payoff is, according to Player $i$,

$$
R^{i}\left(\tau_{1}, \tau_{2}\right):=\mathbb{E}^{i}\left[e^{-r_{i}\left(\tau_{1} \wedge \tau_{2}\right)}\left(G_{1}\left(X_{\tau_{1}}\right) 1_{\left\{\tau_{1} \leq \tau_{2}\right\}}+G_{2}\left(X_{\tau_{2}}\right) 1_{\left\{\tau_{2}<\tau_{1}\right\}}\right)\right]
$$

where $\mathbb{E}^{i}$ denotes the expectation under the measure $\mathbb{P}^{i}$. Naturally, if Player 2 chooses the stopping time $\tau_{2}$, then the aim of Player 1 is to find a stopping time $\tau_{1}^{*}$ such that

$$
R^{1}\left(\tau_{1}^{*}, \tau_{2}\right)=\sup _{\tau_{1}} R^{1}\left(\tau_{1}, \tau_{2}\right) .
$$

Analogously, if Player 1 chooses the stopping time $\tau_{1}$, then Player 2 aims at finding a stopping time $\tau_{2}^{*}$ such that

$$
R^{2}\left(\tau_{1}, \tau_{2}^{*}\right)=\inf _{\tau_{2}} R^{2}\left(\tau_{1}, \tau_{2}\right) .
$$

Definition 2.1. A pair of stopping times $\left(\tau_{1}^{*}, \tau_{2}^{*}\right)$ is a Nash equilibrium if

$$
R^{1}\left(\tau_{1}, \tau_{2}^{*}\right) \leq R^{1}\left(\tau_{1}^{*}, \tau_{2}^{*}\right)
$$

and

$$
R^{2}\left(\tau_{1}^{*}, \tau_{2}\right) \geq R^{2}\left(\tau_{1}^{*}, \tau_{2}^{*}\right)
$$

for any stopping times $\tau_{1}$ and $\tau_{2}$. We denote by $N E$ the set of Nash equilibria.

The process $Z$, defined by (1), defines the change of measure from $\mathbb{P}^{1}$ to $\mathbb{P}^{2}$ by

$$
\mathbb{P}^{2}(A)=\mathbb{E}^{1}\left(Z_{T} 1_{A}\right) \quad \forall A \in \mathcal{F}_{T} .
$$

This allows us to transform the zero-sum game under heterogeneous beliefs into a nonzero-sum game under homogeneous beliefs, and thus the main result from [10] may be employed to guarantee the existence of a Nash equilibrium.

Theorem 2.2. Assume that the processes

$$
\begin{gathered}
X_{t}^{1}:=e^{-r_{1} t} G_{1}\left(X_{t}\right), \\
X_{t}^{2}:=-e^{-r_{2} t} G_{2}\left(X_{t}\right) Z_{t}, \\
Y_{t}^{1}:=e^{-r_{1} t} G_{2}\left(X_{t}\right)
\end{gathered}
$$


and

$$
Y_{t}^{2}:=-e^{-r_{2} t} G_{1}\left(X_{t}\right) Z_{t}
$$

are uniformly integrable with respect to $\mathbb{P}^{1}$, and that $\lim _{t \rightarrow \infty} X_{t}^{i}=\lim _{t \rightarrow \infty} Y_{t}^{i}=$ $0 \mathbb{P}^{1}$-a.s., $i=1,2$. Then the set $N E$ is non-empty.

Proof. First note that

$$
\begin{aligned}
R^{2}\left(\tau_{1}, \tau_{2}\right) & =\mathbb{E}^{2}\left[e^{-r_{2}\left(\tau_{1} \wedge \tau_{2}\right)}\left(G_{1}\left(X_{\tau_{1}}\right) 1_{\left\{\tau_{1} \leq \tau_{2}\right\}}+G_{2}\left(X_{\tau_{2}}\right) 1_{\left\{\tau_{2}<\tau_{1}\right\}}\right)\right] \\
& =\mathbb{E}^{1}\left[e^{-r_{2}\left(\tau_{1} \wedge \tau_{2}\right)} Z_{\tau_{1} \wedge \tau_{2}}\left(G_{1}\left(X_{\tau_{1}}\right) 1_{\left\{\tau_{1} \leq \tau_{2}\right\}}+G_{2}\left(X_{\tau_{2}}\right) 1_{\left\{\tau_{2}<\tau_{1}\right\}}\right)\right] .
\end{aligned}
$$

Thus the zero-sum game under heterogeneous beliefs may be written as a nonzero-sum games under homogeneous beliefs. Under the measure $\mathbb{P}^{1}$, the corresponding processes $e^{-r_{1} t} G_{1}\left(X_{t}\right),-e^{-r_{2} t} G_{2}\left(X_{t}\right) Z_{t}, e^{-r_{1} t} G_{2}\left(X_{t}\right)$ and $-e^{-r_{2} t} G_{1}\left(X_{t}\right) Z_{t}$ satisfy the conditions A1-A3 from [10], and [10, Theorem 2.2] thus applies (the result in [10] is formulated for a finite time horizon, but it extends to the present setting of infinite horizon with uniformly integrable payoff processes).

Remark The connection with nonzero-sum games makes it possible to use results from the theory of such games. In particular, it gives the existence of a Nash equilibrium. However, there seems to be little known about exact conditions for uniqueness of equilibria for nonzero-sum stopping games. Moreover, note that even though we have formulated the zero-sum game with heterogeneous beliefs in a Markovian setting, the resulting nonzerosum game with homogeneous beliefs is non-Markovian, thus making, for example, the results of [3] not directly applicable.

To a given Nash equilibrium $\left(\tau_{1}^{*}, \tau_{2}^{*}\right)$ we associate the corresponding value

$$
V_{1}^{\left(\tau_{1}^{*}, \tau_{2}^{*}\right)}=\sup _{\tau_{1}} R^{1}\left(\tau_{1}, \tau_{2}^{*}\right)=R^{1}\left(\tau_{1}^{*}, \tau_{2}^{*}\right)
$$

for Player 1 and the value

$$
V_{2}^{\left(\tau_{1}^{*}, \tau_{2}^{*}\right)}=\inf _{\tau_{2}} R^{2}\left(\tau_{1}^{*}, \tau_{2}\right)=R^{2}\left(\tau_{1}^{*}, \tau_{2}^{*}\right)
$$

for Player 2. In the case when the players agree about the model parameters, i.e. when $\mathbb{P}^{1}=\mathbb{P}^{2}$ and $r_{1}=r_{2}$, the existence of a Nash equilibrium $\left(\tau_{1}^{*}, \tau_{2}^{*}\right)$ implies that

$$
\begin{aligned}
\bar{V} & :=\inf _{\tau_{2}} \sup _{\tau_{1}} R^{2}\left(\tau_{1}, \tau_{2}\right)=\inf _{\tau_{2}} \sup _{\tau_{1}} R^{1}\left(\tau_{1}, \tau_{2}\right) \leq \sup _{\tau_{1}} R^{1}\left(\tau_{1}, \tau_{2}^{*}\right) \\
& =R^{1}\left(\tau_{1}^{*}, \tau_{2}^{*}\right)=R^{2}\left(\tau_{1}^{*}, \tau_{2}^{*}\right)=\inf _{\tau_{2}} R^{2}\left(\tau_{1}^{*}, \tau_{2}\right) \\
& \leq \sup _{\tau_{1}} \inf _{\tau_{2}} R^{2}\left(\tau_{1}, \tau_{2}\right) \leq \bar{V} .
\end{aligned}
$$

Consequently, the value for Player 1 and the value for Player 2 agree, and this common value does not depend on the Nash equilibrium in the sense that if several Nash equilibria exist, then all the corresponding associated values coincide. On the other hand, under heterogeneity, different Nash 
equilibria may have different associated values. In fact, this is the case for the game version of the American call option, see Section 4 below.

\section{A VERIFICATION RESUlT}

In this section we provide a verification result that shows that if a given pair of functions $\left(U_{1}(x), U_{2}(x)\right)$ satisfies a set of natural conditions, then a Nash equilibrium of stopping times is obtained such that $\left(U_{1}(x), U_{2}(x)\right)$ is the corresponding pair of values.

Let $U_{i}: \mathbb{R} \rightarrow \mathbb{R}, i=1,2$, be continuous functions such that

$$
U_{1} \geq G_{1}
$$

and

$$
U_{2} \leq G_{2}
$$

Also, denote the stopping region of Player $i, i=1,2$, by

$$
\mathcal{D}_{i}:=\left\{x \in \mathbb{R}: U_{i}(x)=G_{i}(x)\right\},
$$

and denote by

$$
\tau_{\mathcal{A}}:=\inf \left\{t \geq 0: X_{t} \in \mathcal{A}\right\}
$$

the first hitting time of a closed set $\mathcal{A}$. For ease of notation, if $\mathcal{A}=\{a\}$, i.e. a singleton, then we write $\tau_{a}$ instead of $\tau_{\{a\}}$.

Theorem 3.1. Assume that

(i) the functions $U_{i}, i=1,2$ are continuous and satisfy (3) and (4);

(ii) $\left\{e^{-r_{1}\left(t \wedge \tau_{\mathcal{D}_{2}}\right)} U_{1}\left(X_{t \wedge \tau_{\mathcal{D}_{2}}}\right), 0 \leq t \leq \infty\right\}$ is a $\mathbb{P}^{1}$-supermartingale;

(iii) $\left\{e^{-r_{2}\left(t \wedge \tau_{\mathcal{D}_{1}}\right)} U_{2}\left(X_{t \wedge \tau_{\mathcal{D}_{1}}}\right), 0 \leq t \leq \infty\right\}$ is a $\mathbb{P}^{2}$-submartingale;

(iv) $\left\{e^{-r_{i}\left(t \wedge \tau_{\mathcal{D}_{1}} \wedge \tau_{\mathcal{D}_{2}}\right)} U_{i}\left(X_{t \wedge \tau_{\mathcal{D}_{1}} \wedge \tau_{\mathcal{D}_{2}}}\right), 0 \leq t \leq \infty\right\}$ is a $\mathbb{P}^{i}$-martingale, $i=$ 1,2 ;

(v) $U_{1} \equiv U_{2}$ on $\mathcal{D}_{1} \cup \mathcal{D}_{2}$.

Then $\left(\tau_{\mathcal{D}_{1}}, \tau_{\mathcal{D}_{2}}\right)$ is a Nash equilibrium of stopping times. Moreover, $U_{1}$ is the corresponding value for Player 1 and $U_{2}$ is the corresponding value for Player 2.

Remark One way to produce candidate functions $U_{1}$ and $U_{2}$ is by solving the coupled variational inequalities

$$
\left\{\begin{array}{l}
\max \left\{\mathcal{L}_{1} U_{1}, G_{1}-U_{1}\right\}=0 \text { on }\left\{U_{2}<G_{2}\right\} \\
\min \left\{\mathcal{L}_{2} U_{2}, G_{2}-U_{2}\right\}=0 \text { on }\left\{U_{1}>G_{1}\right\}
\end{array}\right.
$$

where the differential operators $\mathcal{L}_{i}, i=1,2$, are defined by

$$
\mathcal{L}_{i}:=\frac{1}{2} \sigma^{2} \frac{d^{2}}{d x^{2}}+\mu_{i} \frac{d}{d x}-r_{i} .
$$

However, note that conditions (ii)-(iv) involve martingale properties up to (and including) time infinity, where, by convention, the processes equal zero. Thus additional care is needed when checking that a solution of the coupled variational inequalities indeed provides a Nash equilibrium. 
Proof. From assumption (ii) and the optional sampling theorem we have

$$
\mathbb{E}^{1}\left[e^{-r_{1}\left(\tau_{1} \wedge \tau_{\mathcal{D}_{2}}\right)} U_{1}\left(X_{\tau_{1} \wedge \tau_{\mathcal{D}_{2}}}\right)\right] \leq U_{1}(x)
$$

for any stopping time $\tau_{1}$. Since $U_{1} \geq G_{1}$ and $U_{1}\left(X_{\tau_{\mathcal{D}_{2}}}\right)=G_{2}\left(X_{\tau_{\mathcal{D}_{2}}}\right)$, we have

$$
\begin{aligned}
& \mathbb{E}^{1}\left[e^{-r_{1}\left(\tau_{1} \wedge \tau_{\mathcal{D}_{2}}\right)} U_{1}\left(X_{\tau_{1} \wedge \tau_{\mathcal{D}_{2}}}\right)\right] \\
& \geq \mathbb{E}^{1}\left[e^{-r_{1}\left(\tau_{1} \wedge \tau_{\mathcal{D}_{2}}\right)}\left(G_{1}\left(X_{\tau_{1}}\right) 1_{\left\{\tau_{1} \leq \tau_{\mathcal{D}_{2}}\right\}}+G_{2}\left(X_{\tau_{\mathcal{D}_{2}}}\right) 1_{\left\{\tau_{\mathcal{D}_{2}}<\tau_{1}\right\}}\right)\right] \\
& =R^{1}\left(\tau_{1}, \tau_{\mathcal{D}_{2}}\right) \text {. }
\end{aligned}
$$

Thus

$$
R^{1}\left(\tau_{1}, \tau_{\mathcal{D}_{2}}\right) \leq U_{1}(x)
$$

so taking the supremum over stopping times $\tau_{1}$ yields

$$
\sup _{\tau_{1}} R^{1}\left(\tau_{1}, \tau_{\mathcal{D}_{2}}\right) \leq U_{1}(x) .
$$

Similarly, (iii) yields

$$
U_{2}(x) \leq \mathbb{E}^{2}\left[e^{-r_{2}\left(\tau_{\mathcal{D}_{1}} \wedge \tau_{2}\right)} U_{2}\left(X_{\tau_{\mathcal{D}_{1}} \wedge \tau_{2}}\right)\right]
$$

for any stopping time $\tau_{2}$. Since $U_{2} \leq G_{2}$ and $U_{2}\left(X_{\tau_{\mathcal{D}_{1}}}\right)=G_{1}\left(X_{\tau_{\mathcal{D}_{1}}}\right)$, we have

$$
\begin{aligned}
\mathbb{E}^{2}\left[e^{-r_{2}\left(\tau_{\mathcal{D}_{1}} \wedge \tau_{2}\right)} U_{2}\left(X_{\mathcal{T}_{1} \wedge \tau_{2}}\right)\right] \\
\quad \leq \mathbb{E}^{2}\left[e^{-r_{2}\left(\tau_{\mathcal{D}_{1}} \wedge \tau_{2}\right)}\left(G_{1}\left(X_{\tau_{\mathcal{D}_{1}}}\right) 1_{\left\{\tau_{\mathcal{D}_{1}} \leq \tau_{2}\right\}}+G_{2}\left(X_{\tau_{2}}\right) 1_{\left\{\tau_{2}<\tau_{\mathcal{D}_{1}}\right\}}\right)\right] \\
\quad=R^{2}\left(\tau_{\mathcal{D}_{1}}, \tau_{2}\right) .
\end{aligned}
$$

Thus

$$
U_{2}(x) \leq R^{2}\left(\tau_{\mathcal{D}_{1}}, \tau_{2}\right),
$$

so taking the infimum over stopping times $\tau_{2}$ yields

$$
U_{2}(x) \leq \inf _{\tau_{2}} R^{2}\left(\tau_{\mathcal{D}_{1}}, \tau_{2}\right) .
$$

Finally, (iv) gives

$$
\mathbb{E}^{i}\left[e^{-r_{i}\left(\tau_{\mathcal{D}_{1}} \wedge \tau_{\mathcal{D}_{2}}\right)} U_{i}\left(X_{\tau_{\mathcal{D}_{1}} \wedge \tau_{\mathcal{D}_{2}}}\right)\right]=U_{i}(x)
$$

for $i=1,2$. Since $U_{i}\left(X_{\tau_{\mathcal{D}_{1}}}\right)=G_{1}\left(X_{\tau_{\mathcal{D}_{1}}}\right)$ and $U_{i}\left(X_{\tau_{\mathcal{D}_{2}}}\right)=G_{2}\left(X_{\tau_{\mathcal{D}_{2}}}\right)$, we have that

$$
\begin{aligned}
& \mathbb{E}^{i}\left[e^{-r_{i}\left(\tau_{\mathcal{D}_{1}} \wedge \tau_{\mathcal{D}_{2}}\right)} U_{i}\left(X_{\tau_{\mathcal{D}_{1}} \wedge \tau_{\mathcal{D}_{2}}}\right)\right] \\
& =\mathbb{E}^{i}\left[e^{-r_{i}\left(\tau_{\mathcal{D}_{1}} \wedge \tau_{\mathcal{D}_{2}}\right)}\left(G_{1}\left(X_{\tau_{\mathcal{D}_{1}}}\right) 1_{\left\{\tau_{\mathcal{D}_{1}} \leq \tau_{\mathcal{D}_{2}}\right\}}+G_{2}\left(X_{\tau_{\mathcal{D}_{2}}}\right) 1_{\left\{\tau_{\mathcal{D}_{2}}<\tau_{\mathcal{D}_{1}}\right\}}\right)\right] \\
& =R^{i}\left(\tau_{\mathcal{D}_{1}}, \tau_{\mathcal{D}_{2}}\right) .
\end{aligned}
$$

Hence,

$$
U_{i}(x)=R^{i}\left(\tau_{\mathcal{D}_{1}}, \tau_{\mathcal{D}_{2}}\right)
$$


for $i=1,2$, which shows that the inequalities in (6) and (7) are in fact equalities. Consequently, $\left(\tau_{\mathcal{D}_{1}}, \tau_{\mathcal{D}_{2}}\right)$ is a Nash equilibrium of stopping times, and $U_{1}$ and $U_{2}$ are the corresponding value functions.

\section{The game Version of AN AMERICAN CALL Option}

In this section we study the game version of an American call option for a geometric Brownian motion under heterogeneous beliefs. By a logarithmic change of variables, it is clear that the results of Sections 2-3 above (formulated for processes on the whole real line) apply also to this example. For the convenience of the reader we first recall from [19] (see also [7]) the results under homogeneous beliefs.

4.1. Homogeneous beliefs. In this subsection we assume that $G_{1}(x)=$ $(x-K)^{+}$and $G_{2}(x)=G_{1}(x)+\delta$ for some constants $0<\delta<K$. We also assume that $r_{1}=r_{2}=: r$, and that $\mu_{1}(x)=\mu_{2}(x)=\mu x$ for some constant $\mu$ and (by abuse of notation) $\sigma(x)=\sigma x$ for some constant $\sigma>0$. Now $\mathbb{P}^{1}$ and $\mathbb{P}^{2}$ coincide, and we denote it by $\mathbb{P}$. In this case, the value function and the optimal strategies are determined in [6] if $\mu=r$ and in [19] for $\mu<r$.

For $\mu<r$, denote by

$$
v_{A}(x, \mu)=\sup _{\tau} \mathbb{E}\left[e^{-r \tau}\left(X_{\tau}-K\right)^{+}\right]
$$

the value function of an American call option for a drift $\mu$, and denote by

$$
v_{B}(x, \mu)=\sup _{\tau} \mathbb{E}\left[e^{-r\left(\tau \wedge \tau_{K}\right)}\left(\left(X_{\tau}-K\right)^{+} 1_{\left\{\tau<\tau_{K}\right\}}+\delta 1_{\left\{\tau \geq \tau_{K}\right\}}\right)\right]
$$

the value function of an American down-and-out call option with rebate $\delta$ at the barrier $K$. Let $c$ be the solution to

$$
v_{A}(K, c)=\delta,
$$

and let $d$ be the solution to

$$
\frac{\partial^{+} v_{B}}{\partial x}(K, d)=1
$$

where $\frac{\partial^{+}}{\partial x}$ denotes the right derivative. Furthermore, let

$$
A:=\inf \left\{x \geq K: v_{A}(x, \mu)=G_{1}(x)\right\}
$$

be the optimal stopping boundary for an American call with drift $\mu$, and let

$$
B:=\inf \left\{x \geq K: v_{B}(x, \mu)=G_{1}(x)\right\}
$$

be the optimal stopping boundary for an American down-and-out call option with rebate $\delta$ at $K$.

Theorem 4.1. (Yam, Yung, Zhou [19]) Assume that $\delta<K$. Then the constants $c$ and $d$ are well-defined and satisfy $c<d<r$. Denote by $V$ the value function.

- If $\mu<c$, then $V(x)=v_{A}(x, \mu)$.

- If $c \leq \mu \leq d$, then $V(x)=v_{B}(x, \mu)$. 
- If $d<\mu<r$, then

$$
V(x)= \begin{cases}G_{2}(x) & \text { for } x \in\left[K, b_{1}\right] \\ G_{1}(x) & \text { for } x \in\left[b_{2}, \infty\right)\end{cases}
$$

and $V$ solves $\frac{1}{2} \sigma^{2} x^{2} \frac{\partial^{2} V}{\partial x^{2}}+\mu x \frac{\partial V}{\partial x}-r V=0$ on $(0, K)$ and on $\left(b_{1}, b_{2}\right)$ with the obvious boundary conditions. Here $b_{1}$ and $b_{2}$ are found by solving a coupled pair of equations originating from the smooth-fit condition.

Moreover, the pair $\left({\mathcal{\mathcal { D } _ { 1 }}}_{1}, \tau_{\mathcal{D}_{2}}\right)$ is a Nash equilibrium, where $\mathcal{D}_{1}=\{x: V(x)=$ $\left.G_{1}(x)\right\}$ and $\mathcal{D}_{2}=\left\{x: V(x)=G_{2}(x)\right\}$.

Remark Note that in the case when $\mu=c$, we have $v_{A}(x, c)=v_{B}(x, c)$, and both $\left(\tau_{\mathcal{D}_{1}}, \tau_{\mathcal{D}_{2}}\right)$ and $\left(\tau_{\mathcal{D}_{1}}, \infty\right)$ are Nash equilibria. Also note that if $\mu=r$, then the process $e^{-r t} G_{2}\left(X_{t}\right)$ is not uniformly integrable, so Theorem 2.2 does not apply. In fact, in this case there is no Nash equilibrium, compare $[6]$.

4.2. Heterogeneous beliefs. For simplicity, we assume that $r_{1}=r_{2}$, and we denote this common value by $r$, so heterogeneity only applies to the drift of $X$. Let $G_{1}$ and $G_{2}$ be defined as above, and assume that (by abuse of notation) $\mu_{i}(x)=\mu_{i} x$ and $\sigma(x)=\sigma x$ for constants $\mu_{1}, \mu_{2}$ and $\sigma>0$. Since it is natural that the buyer of a call option estimates the drift higher than does the seller, we restrict our attention to the case $\mu_{2}<\mu_{1}<r$. The case $\mu_{1}<\mu_{2}$ can be treated similarly and hence this is a rather mild restriction. We divide the analysis into three separate cases, depending on the value of $\mu_{1}$. Furthermore, to illustrate our results graphically we choose a set of base-case parameters to be $\sigma=0.15, r=0.05, K=1$, and $\delta=0.1$. Given these values we can calculate that $c \approx-0.024$ and $d \approx 0.018$.

For $\mu \in \mathbb{R}$, denote by $\mathbb{P}_{\mu}$ a measure under which $X$ has drift $\mu$. The function

$$
H(x, \mu, \mathcal{A}, \mathcal{B}):=\mathbb{E}_{\mu}\left[e^{-r\left(\tau_{\mathcal{A}} \wedge \tau_{\mathcal{B}}\right)}\left(G_{1}\left(X_{\tau_{\mathcal{A}}}\right) 1_{\left\{\tau_{\mathcal{A}} \leq \tau_{\mathcal{B}}\right\}}+G_{2}\left(X_{\tau_{\mathcal{B}}}\right) 1_{\left\{\tau_{\mathcal{B}}<\tau_{\mathcal{A}}\right\}}\right)\right]
$$

is used in the subsections below. Note that $H$ represents the value of the game, evaluated by an agent with drift $\mu$, provided that Player 1 stops the first instant that the process $X$ enters $\mathcal{A}$ and Player 2 stops when $X$ enters $\mathcal{B}$.

\subsubsection{The case $\mu_{2}<\mu_{1} \leq c$.}

Theorem 4.2. If $\mu_{2}<\mu_{1} \leq c$, then the pair $\left(\tau_{[A, \infty)}, \infty\right)$, where $A$ is defined by (8) with $\mu=\mu_{1}$, is a Nash equilibrium.

Proof. It is straightforward to check that the pair $\left(U_{1}, U_{2}\right)$, where $U_{1}:=$ $v_{A}\left(x, \mu_{1}\right)$ and $U_{2}:=H\left(x, \mu_{2},[A, \infty), \emptyset\right)$, satisfies the assumptions of Theorem 3.1. Therefore, the result follows.

The value functions of the Nash equilibrium described in Theorem 4.2 are illustrated in Figure 1. 


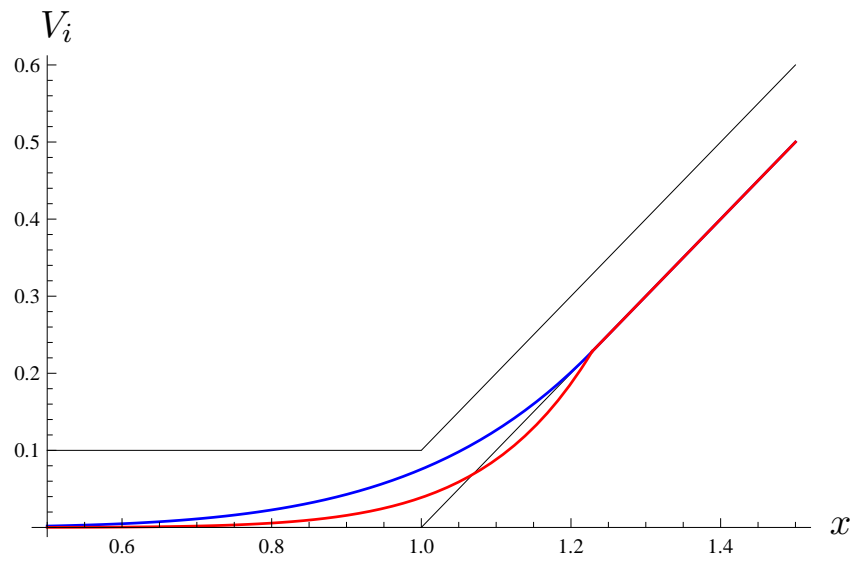

Figure 1 . The case $\mu_{2}<\mu_{1} \leq c$. The parameter values used above are $\mu_{2}=-0.08<\mu_{1}=-0.04<c$. For these parameters, $A \approx 1.23$, and the value of Player 1 (blue) is above the value of Player 2 (red).

4.2.2. The case $\mu_{2}<\mu_{1} \in(c, d)$. Let $A$ and $B$ be defined as in (8) and (9) with $\mu=\mu_{1}$, respectively. Since $\mu_{1}>c$, it is straightforward to check that $B<A$. Let $m_{1}$ and $m_{2}$ be the unique solutions of

$$
H\left(K, m_{1},[B, \infty), \emptyset\right)=\delta
$$

and

$$
H\left(K, m_{2},[A, \infty), \emptyset\right)=\delta,
$$

respectively. In words, if Player 1 stops at $B$ (i.e. the best response if Player 2 stops at $K$ ), and Player 2 never stops, then $m_{1}$ is the drift that makes the value for Player 2 equal to $\delta$ at $x=K$. Similarly, if Player 1 stops at $A$ (i.e. the best response if Player 2 never stops), and Player 2 never stops, then $m_{2}$ is the drift that makes the value for Player 2 equal to $\delta$ at $x=K$. Since $H(K, \mu,[B, \infty), \emptyset)$ and $H(K, \mu,[A, \infty), \emptyset)$ are strictly increasing in $\mu$, the thresholds $m_{1}$ and $m_{2}$ are well-defined. Moreover, they satisfy $m_{1}<\mu_{1}$ and $m_{2}<\mu_{1}$.

\section{Theorem 4.3.}

(i) If $\mu_{2} \in\left(-\infty, m_{2}\right]$, then $\left(\tau_{[A, \infty)}, \infty\right)$ is a Nash equilibrium.

(ii) If $\mu_{2} \in\left[m_{1}, \mu_{1}\right)$, then $\left(\tau_{[B, \infty)}, \tau_{K}\right)$ is a Nash equilibrium.

Furthermore, the inequality $m_{1} \leq m_{2}$ holds.

Proof. First assume that $\mu_{2} \in\left(-\infty, m_{2}\right]$. Let $U_{1}(x):=v_{A}\left(x, \mu_{1}\right)$, and define

$$
U_{2}(x):=H\left(x, \mu_{2},[A, \infty), \emptyset\right) .
$$

Since $\mu_{2} \leq m_{2}$, we have $U_{2} \leq G_{2}$ everywhere. Moreover, it is straightforward to check that $U_{1}$ and $U_{2}$ satisfy the remaining conditions of Theorem 3.1, so $\left(\tau_{[A, \infty)}, \infty\right)$ is a Nash equilibrium. 
Next assume that $\mu_{2} \in\left[m_{1}, \mu_{1}\right)$. Let $U_{1}(x):=v_{B}\left(x, \mu_{1}\right)$, and define

$$
U_{2}(x):=H\left(x, \mu_{2},[B, \infty),\{K\}\right),
$$

i.e. the value for Player 2 if Player 1 stops at $B$ and Player 2 stops at $K$. Then $U_{1}$ and $U_{2}$ satisfy the conditions of Theorem 3.1 , so $\left(\tau_{[B, \infty)}, \tau_{K}\right)$ is a Nash equilibrium.

Finally note that the function $h(x):=H\left(x, m_{2},[A, \infty), \emptyset\right)$ is convex and satisfies $h(K)=\delta$ and $\frac{\partial^{-} h}{\partial x}(A)>1$. Consequently, there exists a unique point $\hat{B}>K$ such that $h<G_{1}$ on $(\hat{B}, A)$ and $h>G_{1}$ on $(0, \hat{B})$. Moreover, $\hat{\mathcal{L}}_{2} h=0$ on $(0, A)$, where $\hat{\mathcal{L}}_{2}$ is defined as in (5) but with drift $m_{2} x$. Thus $\mathcal{L}_{1} h \geq 0$ on $(K, \hat{B})$ since $h$ is increasing and $m_{2} \leq \mu_{1}$. Consequently, $v_{B}\left(\cdot, \mu_{1}\right) \geq h$ on $(K, \hat{B})$, which shows that $B \geq \hat{B}$. Therefore, $H\left(x, m_{2},[B, \infty), \emptyset\right) \geq H\left(x, m_{2},[A, \infty), \emptyset\right)$ on $(0, B]$, so $m_{1} \leq m_{2}$.

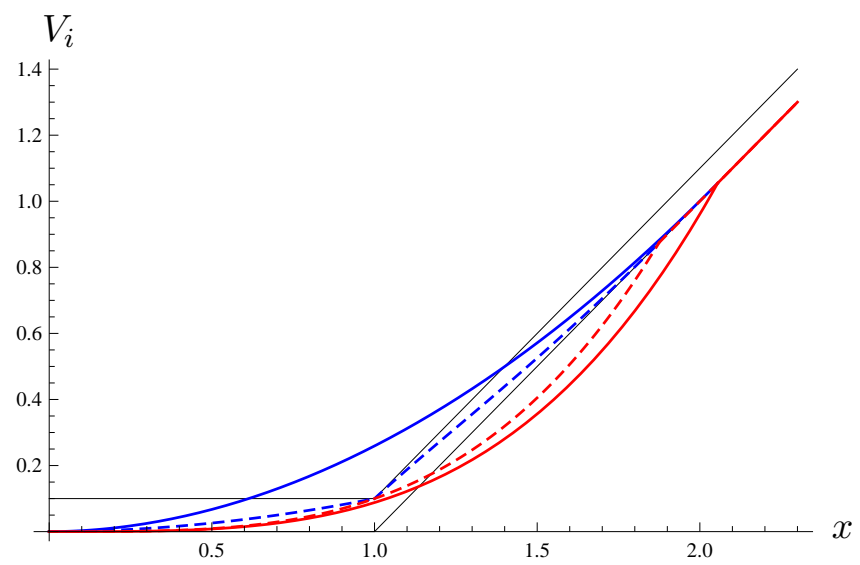

Figure 2. The case $\mu_{2}<\mu_{1} \in(c, d), \mu_{2} \in\left[m_{1}, m_{2}\right]$. The parameter values used above are $\mu_{2}=-0.012<\mu_{1}=0.015 \in$ $(c, d)$. For these parameters we have $m_{1} \approx-0.013, m_{2} \approx$ $-0.010, A \approx 2.05$ and $B \approx 1.88$. The solid lines represent values associated with the Nash equilibrium $\left(\tau_{[A, \infty)}, \infty\right)$ and the dashed lines correspond to the equilibrium $\left(\tau_{[B, \infty)}, \tau_{K}\right)$. The value for Player 1 (blue) is in both cases higher than the corresponding value for Player 2 (red).

Remark By Theorem 4.3, the interval $\left[m_{1}, m_{2}\right]$ is non-empty (actually, a closer inspection of the proof above reveals that $m_{1}<m_{2}$ ). Consequently, if $\mu_{2} \in\left[m_{1}, m_{2}\right]$, then there are multiple Nash equilibria.

Theorem 4.4. Assume that $\mu_{2} \in\left[m_{1}, m_{2}\right]$ so that both $\left(\tau_{[A, \infty)}, \infty\right)$ and $\left(\tau_{[B, \infty)}, \tau_{K}\right)$ are Nash equilibria. Then

$$
V_{2}^{\left(\tau_{[A, \infty)}, \infty\right)} \leq V_{2}^{\left(\tau_{[B, \infty)}, \tau_{K}\right)} \leq V_{1}^{\left(\tau_{[B, \infty)}, \tau_{K}\right)} \leq V_{1}^{\left(\tau_{[A, \infty)}, \infty\right)} .
$$


Proof. Let $\mu_{2} \in\left[m_{1}, m_{2}\right]$. Since $v_{B}\left(\cdot, \mu_{1}\right)$ is increasing, we find that $h(x):=$ $H\left(x, \mu_{2},[B, \infty),\{K\}\right)$ satisfies $\mathcal{L}_{1} h \geq \mathcal{L}_{1} v_{B}=0$ on $(0, K) \cup(K, B)$. Consequently, a maximum principle argument shows that $h \leq v_{B}\left(\cdot, \mu_{1}\right)$, which is the second inequality in (10).

Next, the function $h(x):=H\left(x, \mu_{2},[A, \infty), \emptyset\right)$ is convex and satisfies $h(K) \leq \delta$ and $\frac{\partial^{-} h}{\partial x}(A)>1$. Consequently, there exists a unique point $\hat{B}>K$ such that $h<G_{1}$ on $(\hat{B}, A)$ and $h>G_{1}$ on $(0, \hat{B})$. A similar argument as in the proof of Theorem 4.3 (but with drift $\mu_{2}$ instead of $m_{2}$ ) shows that $\hat{B} \leq B$. Therefore, the first inequality in (10) follows from $h(K) \leq \delta$ and $h(B) \leq G_{1}(B)$, and the third inequality follows from $\delta \leq H\left(K, \mu_{1},[A, \infty), \emptyset\right)$ and $G_{1}(B) \leq H\left(B, \mu_{1},[A, \infty), \emptyset\right)$.

The value functions of the Nash equilibria described in Theorems 4.3 and 4.4 are illustrated in Figure 2. By Theorem 4.4, both players prefer the Nash equilibrium $\left(\tau_{[A, \infty)}, \infty\right)$ to $\left(\tau_{[B, \infty)}, \tau_{K}\right)$. In light of this, we refer to a Nash equilibrium as optimal if it is preferred to any other equilibrium by both players. Specifying conditions which guarantee the existence of an optimal equilibrium is an interesting open question.

4.2.3. The case $\mu_{2}<\mu_{1} \in[d, r)$. From the point of view of non-uniqueness of Nash equilibria, this case resembles the one studied in Section 4.2.2 above.

Let $v_{A}\left(x, \mu_{1}\right), v_{B}\left(x, \mu_{1}\right), A, B, m_{1}$ and $m_{2}$ be defined as before. Moreover, let $m_{3}$ be the unique solution of

$$
\frac{\partial^{+} H}{\partial x}\left(K, m_{3},[B, \infty),\{K\}\right)=1 .
$$

Theorem 4.5. We have $m_{1} \leq m_{2}$ and $m_{1} \leq m_{3}$. Moreover, the following statements hold.

(i) If $\mu_{2} \in\left(-\infty, m_{2}\right]$, then $\left(\tau_{[A, \infty)}, \infty\right)$ is a Nash equilibrium.

(ii) If $\mu_{2} \in\left[m_{1}, m_{3}\right]$, then $\left(\tau_{[B, \infty)}, \tau_{K}\right)$ is a Nash equilibrium.

(iii) If $\mu_{2} \in\left(m_{3}, \mu_{1}\right)$, then $\left(\tau_{\left[B_{1}, \infty\right)}, \tau_{\left[K, B_{2}\right]}\right)$ is a Nash equilibrium, where $B_{i}, i=1,2$ satisfy $K<B_{2}<B_{1}<B$ and solve the following free-boundary problem:

$$
\begin{cases}\mathcal{L}_{1} U_{1}(x)=0 & \text { for } x \in\left(B_{2}, B_{1}\right) \\ \mathcal{L}_{2} U_{2}(x)=0 & \text { for } x \in\left(B_{2}, B_{1}\right) \\ U_{1}^{\prime}\left(B_{1}\right)=1 & \\ U_{2}^{\prime}\left(B_{2}\right)=1 & \\ U_{1}(x)=U_{2}(x)=G_{2}(x) & \text { for } x \in\left[K, B_{2}\right] \\ U_{1}(x)=U_{2}(x)=G_{1}(x) & \text { for } x \geq B_{1} .\end{cases}
$$

Proof. The fact $m_{1} \leq m_{2}$ and properties (i)-(ii) can be proved similarly as in Section 4.2.2. To prove that $m_{1} \leq m_{3}$, note that $h(x):=H\left(x, m_{1},[B, \infty),\{K\}\right)$ satisfies $h(K)=\delta$ with $h^{\prime}(K)<1$. Since $h$ is increasing we find that $h(x) \leq H(x, \mu,[B, \infty),\{K\})$ if $\mu \geq m_{1}$ and $h(x) \geq H(x, \mu,[B, \infty),\{K\})$ if $\mu \leq m_{1}$. Consequently, $m_{1} \leq m_{3}$. 


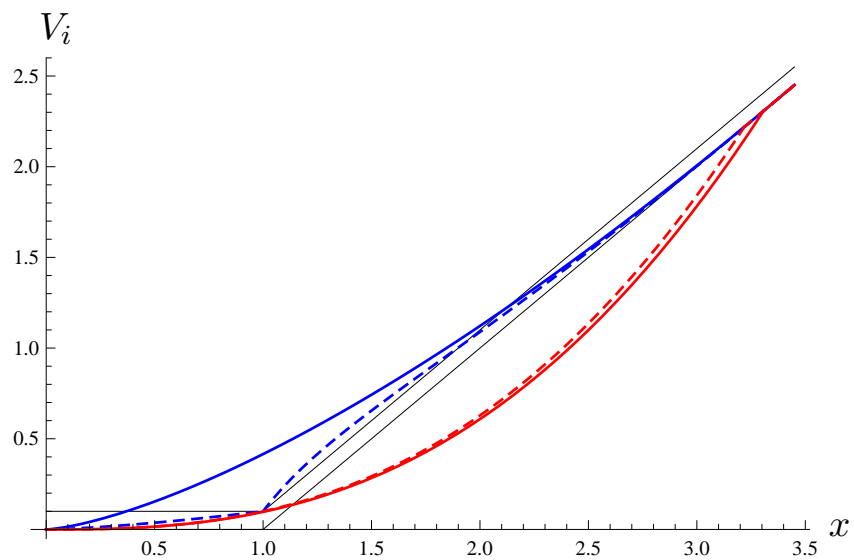

Figure 3 . The case $\mu_{2}<\mu_{1} \in[d, r), \mu_{2} \in\left[m_{1}, m_{2}\right]$. The parameter values used above are $\mu_{2}=0.0003<\mu_{1}=0.03 \in$ $[d, r)$. For these parameters we have $m_{1} \approx 0.00027, m_{2} \approx$ $0.00078, A \approx 3.31$ and $B \approx 3.22$. The solid lines represent values associated with the Nash equilibrium $\left(\tau_{[A, \infty)}, \infty\right)$ and the dashed lines correspond to the equilibrium $\left(\tau_{[B, \infty)}, \tau_{K}\right)$. The value for Player 1 (blue) is in both cases higher than the corresponding value for Player 2 (red).

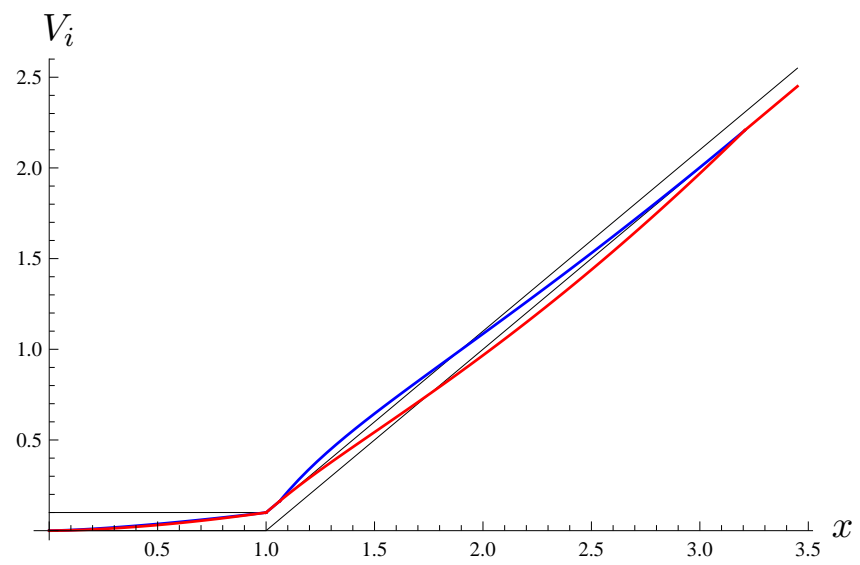

Figure 4 . The case $\mu_{2}<\mu_{1} \in[d, r), \mu_{2} \in\left(m_{3}, \mu_{1}\right)$. The parameter values used above are $\mu_{2}=0.022<\mu_{1}=0.03 \epsilon$ $[d, r)$. For these parameters we have $m_{3} \approx 0.0205, B_{1} \approx 3.21$ and $B_{2} \approx 1.07$. The value for Player 1 (blue) is above the value for Player 2 (red).

The proof of (iii) follows along similar lines as in the proof of [1, Theorem 4.4]. Let $L$ be the unique point above $K$ such that $v_{B}\left(L, \mu_{1}\right)=G_{2}(L)$, 
and let $\tilde{B}$ be defined as the smallest point above $K$ such that

$$
H\left(K, \mu_{2},[\tilde{B}, \infty), \emptyset\right)=\delta .
$$

Then $K<\tilde{B}<B$ and $K<L<B$. Now, given a point $y \in[K, L]$, there exists a unique point $b(y)$ such that if Player 2 uses $\tau_{[K, y]}$, then $\tau_{[b(y), \infty)}$ is the best response for Player 1. Moreover, $b(y) \in[\tilde{B}, B]$, and $b(K)=b(L)=B$. Similarly, given a point $z \in[\tilde{B}, B]$, there exists a unique point $a(z) \geq K$ such that if Player 1 uses $\tau_{[z, \infty)}$, then the best response for Player 2 is $\tau_{[K, a(z)]}$, and $a(B) \in(K, L)$. Thus $a(b(K))-K>0$ and $a(b(L))-L<0$. By continuity of the functions $a$ and $b$, there exists a point $B_{2}$ such that $a\left(b\left(B_{2}\right)\right)-B_{2}=0$. It is then straightforward, using Theorem 3.1, to check that this point $B_{2}$ together with $B_{1}=b\left(B_{2}\right)$ have the desired properties.

Remark We have not been able to establish a relation between $m_{2}$ and $m_{3}$. However, numerical experiments indicate that $m_{2} \leq m_{3}$ at least for a wide range of parameter values.

The value functions of the Nash equilibria described in Theorem 4.5 are illustrated in Figures 3 and 4. Figure 3 shows the multiple equilibria when $\mu_{2} \in\left[m_{1}, m_{2}\right]$ and Figure 4 the equilibrium $\left(\tau_{\left[B_{1}, \infty\right)}, \tau_{\left[K, B_{2}\right]}\right)$ which exists when $\mu_{2} \in\left(m_{3}, \mu_{1}\right)$.

\section{RANDOMized STRATEGIES}

When considering Dynkin games, a natural extension of stopping times (corresponding to pure strategies in game theory) is the concept of randomized stopping times (corresponding to mixed strategies). In this section we study such games under heterogeneous beliefs where the admissible strategies are randomized stopping times, compare [8] and [18]. Our definition of randomized stopping times is equivalent to the one in [18], but the description is slightly changed in order to emphasize an interpretation using intensities of stopping.

To model randomized stopping times, let $\theta$ be a random variable which is exponentially distributed with parameter 1 and independent of $X$. Given an adapted, non-decreasing and right-continuous $[0, \infty]$-valued process $\Gamma$ with $\Gamma_{0-}=0$, define the associated randomized stopping time by

$$
\gamma:=\gamma_{\Gamma}:=\inf \left\{t \geq 0: \Gamma_{t}>\theta\right\}
$$

and denote by

$$
\overline{\mathcal{T}}:=\left\{\gamma_{\Gamma}: \Gamma \text { adapted, non-decreasing, right-continuous with } \Gamma_{0-}=0\right\}
$$

the set of randomized stopping times. In this way, the set of randomized stopping times contains the set of stopping times. Indeed, given a stopping time $\tau$, define

$$
\Gamma_{t}:= \begin{cases}0 & t<\tau \\ \infty & t \geq \tau\end{cases}
$$

Then $\gamma_{\Gamma}=\tau$. 
One natural class of randomized stopping times is the one constructed as follows. Let $\Lambda$ be a non-negative measure on $(0, \infty)$, and define an adapted and non-decreasing process $\tilde{\Gamma}^{\Lambda}$ by

$$
\tilde{\Gamma}_{t}^{\Lambda}:=\int_{\mathbb{R}} \frac{L_{t}(z)}{\sigma^{2}(z)} \Lambda(d z),
$$

where $L_{t}(z)$ is the local time of $X$ at time $t$ at the point $z$. Then $\tilde{\Gamma}$ is left-continuous everywhere, and continuous everywhere but possibly at one time-point, at which it jumps to infinity. The process $\Gamma_{t}^{\Lambda}:=\lim _{s \downarrow t} \tilde{\Gamma}_{s}^{\Lambda}$ is adapted, right-continuous and satisfies $\Gamma_{0-}^{\Lambda}=0$. The corresponding randomized stopping time $\gamma=\gamma_{\Gamma^{\Lambda}}$ is then Markovian in the sense that the intensity of stopping only depends on the current level of $X$.

Example Consider the special case when the measure $\Lambda$ has a continuous density so that $\Lambda(d z)=\lambda(z) d z$ for some continuous function $\lambda$. Then, by the occupation time formula, see [17, Corollary 1 on p. 219],

$$
\Gamma_{t}^{\Lambda}=\int_{0}^{t} \lambda\left(X_{s}\right) d s
$$

Therefore $\lambda(z)$ is the intensity with which $\gamma$ happens given that the current value of $X$ is $z$.

Example As another illustrative example, suppose that $\Lambda=\delta_{x_{0}}$, where $\delta_{x_{0}}$ denotes a point mass at some point $x_{0}$. Then $\Gamma_{t}^{\Lambda}=L_{t}\left(x_{0}\right) / \sigma^{2}\left(x_{0}\right)$, so $\Gamma$ only increases at times when the value of $X$ is $x_{0}$. Thus the corresponding randomized stopping time $\gamma=\gamma_{\Gamma^{\Lambda}}$ satisfies $X_{\gamma}=x_{0}$ on the event $\{\gamma<\infty\}$. Example Finally, assume that $\Lambda=\infty \delta_{x_{0}}$, i.e. an infinite point-mass at $x_{0}$. Then

$$
\Gamma_{t}^{\Lambda}= \begin{cases}0 & t<\tau_{x_{0}} \\ \infty & t \geq \tau_{x_{0}}\end{cases}
$$

Consequently, the corresponding randomized stopping time satisfies $\gamma=\tau_{x_{0}}$.

In order to introduce a random device for each player, we extend the probability space as $\left([0, \infty)^{2} \times \Omega, \lambda_{1} \otimes \lambda_{2} \otimes \mathbb{P}^{1}\right)$, where $\lambda_{1}$ and $\lambda_{2}$ denote the law of independent exponentially distributed random variables $\theta_{1}$ and $\theta_{2}$. By abuse of notation, we use $\mathbb{P}^{i}$ instead of $\lambda_{1} \otimes \lambda_{2} \otimes \mathbb{P}^{i}$.

We may now define Nash equilibria as in Definition 2.1, but where stopping times are replaced by randomized stopping times, and for a given Nash equilibrium there are the corresponding value functions of Player 1 and Player 2, respectively. We then have the following verification result. The proof follows along the same lines as the proof of Theorem 3.1, and is therefore omitted.

Theorem 5.1. Assume that $U_{i}:(0, \infty) \rightarrow[0, \infty), i=1,2$ are continuous functions such that $U_{1} \geq G_{1}$ and $U_{2} \leq G_{2}$. Furthermore, assume that $\left(\gamma_{1}, \gamma_{2}\right)$ is a pair of randomized stopping times such that for any randomized stopping time $\gamma$ we have

(i) $\mathbb{E}^{1}\left[e^{-r_{1}\left(\gamma \wedge \gamma_{2}\right)}\left(U_{1}\left(X_{\gamma}\right) 1_{\left\{\gamma \leq \gamma_{2}\right\}}+G_{2}\left(X_{\gamma_{2}}\right) 1_{\left\{\gamma_{2}<\gamma\right\}}\right)\right] \leq U_{1}(x) ;$ 
(ii) $\mathbb{E}^{2}\left[e^{-r_{2}\left(\gamma \wedge \gamma_{1}\right)}\left(G_{1}\left(X_{\gamma_{1}}\right) 1_{\left\{\gamma_{1} \leq \gamma\right\}}+U_{2}\left(X_{\gamma}\right) 1_{\left\{\gamma<\gamma_{1}\right\}}\right)\right] \geq U_{2}(x)$;

(iii) $\mathbb{E}^{i}\left[e^{-r_{i}\left(\gamma_{1} \wedge \gamma_{2}\right)}\left(G_{1}\left(X_{\gamma_{1}}\right) 1_{\left\{\gamma_{1} \leq \gamma_{2}\right\}}+G_{2}\left(X_{\gamma_{2}}\right) 1_{\left\{\gamma_{2}<\gamma_{1}\right\}}\right)\right]=U_{i}(x), i=$ 1,2 .

Then $\left(\gamma_{1}, \gamma_{2}\right)$ is a Nash equilibrium in randomized stopping times. Moreover, $U_{1}$ and $U_{2}$ are the corresponding values for Player 1 and Player 2, respectively.

We now illustrate Theorem 5.1 in the same example of a game version of the American call option as studied in Section 4.

Example With the notation as in Section 4 above, assume $r_{1}=r_{2}$ and that $\mu_{1}$ and $\mu_{2}$ are such that $\mu_{1} \in(c, d)$ and $m_{1}<\mu_{2}<m_{2}$. For Player 2 we will consider randomized stopping times constructed from point-masses $l \delta_{K}$ at $K$, where $l \in[0, \infty]$, and we will denote the corresponding randomized stopping time by $\gamma_{2}^{l}$.

If $l=0$, then $\gamma_{2}^{l}=\infty$, and the best response for Player 1 is $\gamma_{1}=\tau_{A}$. The corresponding value for Player 2 is therefore $H\left(x, \mu_{2},[A, \infty), \emptyset\right)$, which at $x=K$ satisfies $H\left(K, \mu_{2},[A, \infty), \emptyset\right)<\delta$ since $\mu_{2}<m_{2}$.

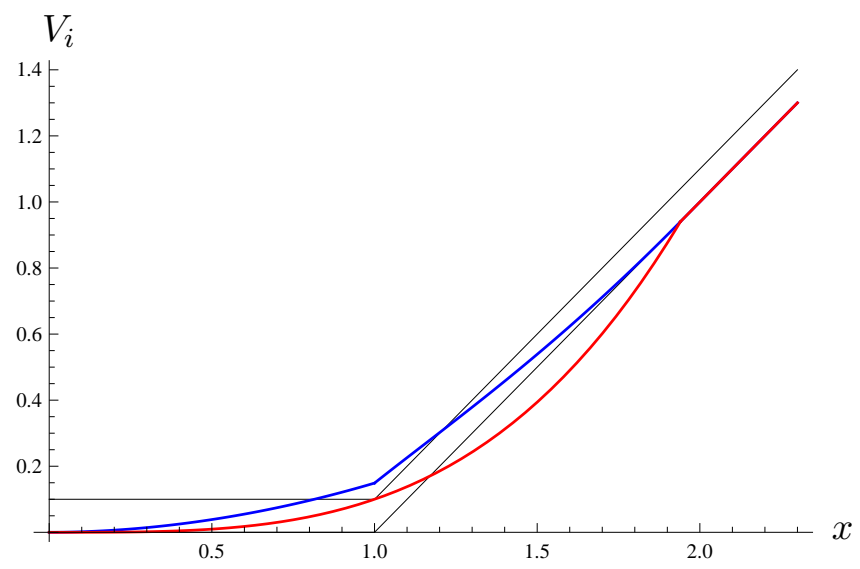

Figure 5. The value functions corresponding to the Nash equilibrium in randomized strategies. The parameters used are the same as in Figure 2. For these parameters we have $l_{0} \approx 0.113$ and $b\left(l_{0}\right) \approx 1.94 \in(B, A)$. Note that the value function for Player 1 (blue) has a kink at $x=K$.

If $l=\infty$, then $\gamma_{2}^{l}=\tau_{K}$, and the best response for Player 1 is $\gamma_{1}=\tau_{B}$. Note that the function $H\left(x, \mu_{2},[B, \infty), \emptyset\right)$ satisfies $H\left(K, \mu_{2},[B, \infty), \emptyset\right)>\delta$ since $\mu_{2}>m_{1}$.

For $l \in(0, \infty)$, the value

$$
U_{1}:=U_{1}^{l}:=\sup _{\gamma_{1} \in \overline{\mathcal{T}}} R^{1}\left(\gamma_{1}, \gamma_{2}^{l}\right)
$$


for Player 1 is given by solving the free-boundary problem

$$
\begin{cases}\mathcal{L}_{1} U_{1}=0 & x \in(0, K) \cup(K, b) \\ \frac{1}{2} \sigma^{2} K^{2}\left(\frac{d^{+}}{d x} U_{1}-\frac{d^{-}}{d x} U_{1}\right)=l\left(U_{1}-\delta\right) & x=K \\ U_{1}(x)=x-K & x \geq b \\ U_{1}^{\prime}(b)=1 . & \end{cases}
$$

Note that $U_{1}$ has a kink at $x=K$, and that the size of the kink is chosen so that the process $e^{-r\left(t \wedge \gamma_{2}^{l}\right)}\left(U_{1}\left(X_{t}\right) 1_{\left\{t \leq \gamma_{2}^{l}\right\}}+G_{2}\left(X_{\gamma_{2}^{l}}\right) 1_{\left\{\gamma_{2}^{l}<t\right\}}\right)$ is a $\mathbb{P}^{1}$ martingale for $t \leq \tau_{b}$. As $l$ increases from 0 to $\infty$, the boundary $b=b(l)$ decreases from $A$ to $B$. By continuity, there exists a value of $l_{0}$ such that $H\left(K, \mu_{2},\left[b\left(l_{0}\right), \infty\right), \emptyset\right)=\delta$. Applying Theorem 5.1 with the candidate functions $U_{1}$ and $U_{2}(x):=H\left(x, \mu_{2},\left[b\left(l_{0}\right), \infty\right), \emptyset\right)$ shows that the pair $\left(\tau_{b\left(l_{0}\right)}, \gamma_{2}^{l_{0}}\right)$ is a Nash equilibrium in randomized stopping times. The corresponding value functions are plotted in Figure 5.

Finally, we note that the equilibrium in randomized strategies is not optimal since, using similar methods as in the proof of Theorem 4.4, it can be shown that

and

$$
V_{1}^{\left(\tau_{[B, \infty)}, \tau_{K}\right)} \leq V_{1}^{\left(\tau_{b\left(l_{0}\right)}, \gamma_{2}^{l_{0}}\right)} \leq V_{1}^{\left(\tau_{[A, \infty)}, \infty\right)}
$$

$$
V_{2}^{\left(\tau_{[A, \infty)}, \infty\right)} \leq V_{2}^{\left(\tau_{b\left(l_{0}\right)}, \gamma_{2}^{l_{0}}\right)} \leq V_{2}^{\left(\tau_{[B, \infty)}, \tau_{K}\right)}
$$

\section{REFERENCES}

[1] Alvarez, L. A class of solvable stopping games. Appl. Math. Optim. 58 (2008), no. 3, 291-314.

[2] Bensoussan, A. and Friedman, A. Nonlinear variational inequalities and differential games with stopping times, J. Funct. Anal., 16 (1974), 305352.

[3] Bensoussan, A. and Friedman, A. Nonzero-sum stochastic differential games with stopping times and free boundary problems. Trans. Amer. Math. Soc. 231 (1977), 275-327.

[4] Dynkin, E.B. Game variant of a problem of optimal stopping, Soviet Math. Dokl., 10 (1969), 16-19.

[5] Ekström, E. and Peskir, G. Optimal stopping games for Markov processes. SIAM J. Control Optim. 47 (2008), no. 2, 684-702.

[6] Ekström, E. and Villeneuve, S. On the value of optimal stopping games. Ann. Appl. Probab. 16 (2006), 1576-1596.

[7] Emmerling, T. Perpetual cancellable American call option. Math. Finance 22 (2012), no. 4, 645-666.

[8] Grün, C. On Dynkin games with incomplete information. SIAM J. Control Optim. 51 (2013), no. 5, 4039-4065.

[9] He, X.-Z. Recent developments in asset pricing with heterogeneous beliefs and adaptive behaviour of financial markets. Global analysis of dynamic models in economics and finance, 3-34, Springer, Heidelberg, 2013. 
[10] Hamadène, S. and Zhang, J. The continuous time nonzero-sum Dynkin game problem and application in game options. SIAM J. Control Optim. 48 (2009/10), no. 5, 3659-3669.

[11] Kurz, M. Rational diverse beliefs and economic volatility In: T. Hens and K. Schenk-Hoppe, eds., Handbook of Financial Markets: Dynamics and Evolution, North Holland, (2008), 439-506.

[12] Karatzas, I. and Shreve, S. Brownian motion and stochastic calculus. Second edition. Graduate Texts in Mathematics, 113. Springer-Verlag, New York, 1991.

[13] Kifer, Y. Game options. Finance Stoch. 4 (2000), no. 4, 443-463.

[14] Kyprianou, A. Some calculations for Israeli options. Finance Stoch. 8 (2004), no. 1, 73-86.

[15] Lempa, J. and Matomäki, P. A Dynkin game with asymmetric information. Stochastics 85 (2013), no. 5, 763-788.

[16] Lepeltier, J.-P. and Maingueneau, M. A. Le jeu de Dynkin en théorie générale sans l'hypothèse de Mokobodski. Stochastics 13 (1984), no. 1-2, 25-44.

[17] Protter, P. Stochastic integration and differential equations. Second edition. Version 2.1.. Stochastic Modelling and Applied Probability, 21. Springer-Verlag, Berlin, 2005.

[18] Touzi, N. and Vieille, N. Continuous-time Dynkin games with mixed strategies. SIAM J. Control Optim. 41 (2002), no. 4, 1073-1088

[19] Yam, S.C.P., Yung, S.P. and Zhou, W. Game call options revisited. Math. Finance 24 (2014), no. 1, 173-206.

Ekström And Leniec: Uppsala University, Box 480, 75106 Uppsala, Sweden.

Glover: University of Technology Sydney, P.O. Box 123, Broadway, NSW 2007, Australia. 